\title{
Self-Stimulated Pulse Echo Trains from Inhomogeneously Broadened Spin Ensembles
}

\author{
Kamanasish Debnath, ${ }^{1, *}$ Gavin Dold $\odot,{ }^{2,3}$ John J. L. Morton, ${ }^{2,4}$ and Klaus Mølmer ${ }^{1}$ \\ ${ }^{1}$ Department of Physics and Astronomy, Aarhus University, Ny Munkegade 120, DK-8000 Aarhus C, Denmark \\ ${ }^{2}$ London Centre for Nanotechnology, University College London, London WC1H OAH, United Kingdom \\ ${ }^{3}$ National Physical Laboratory, Hampton Road, Teddington TW11 OLW, United Kingdom \\ ${ }^{4}$ Department of Electronic and Electrical Engineering, UCL, London WC1E 7JE, United Kingdom
}

(Received 3 April 2020; accepted 6 July 2020; published 22 September 2020)

\begin{abstract}
We show experimentally and describe theoretically how a conventional magnetic resonance Hahn echo sequence can lead to a self-stimulated pulse echo train when an inhomogeneously broadened spin ensemble is coupled to a resonator. Effective strong coupling between the subsystems assures that the first Hahn echo can act as a refocusing pulse on the spins, leading to self-stimulated secondary echoes. Within the framework of mean field theory, we show that this process can continue multiple times leading to a train of echoes. We introduce an analytical model that explains the shape of the first echo and numerical results that account well for the experimentally observed shape and strength of the echo train and provides insights into the collective effects involved.
\end{abstract}

DOI: 10.1103/PhysRevLett.125.137702

Introduction.-Electron spin resonance (ESR) [1,2] and nuclear magnetic resonance (NMR) [3,4] are used in diverse branches of science, ranging from spectroscopic studies in biochemistry and materials science [5-8] to imaging of internal organs in medicine [9]. In NMR and ESR, an ensemble of spins is typically placed within a resonator, controlled by the application of resonant pulses, and measured via emission of signals into a resonator mode. As the spin ensembles are typically inhomogeneous, a common solution is to use control pulses which refocus inhomogeneous interactions, reversing the time evolution of different spin packets to produce a spin echo $[10,11]$ or "Hahn echo" [12]. Aside from being the cornerstone of pulsed NMR and ESR techniques, spin echoes have also become an essential ingredient in quantum information science due to their applications in ensemble quantum memories with ESR and optical transitions [13-17], and in nanoscale quantum metrology $[18,19]$, as well as being a building block for more complex dynamical decoupling sequences used to extend qubit coherence times [20].

The Hahn echo sequence consists of an initial $\pi / 2$ pulse, a time interval $\tau$ and a $\pi$ pulse leading to the emission of a spin echo at time $2 \tau$. The $\pi / 2$ pulse excites the spins into a coherent superposition state, which starts precessing at the Larmor frequencies of the individual spins, leading to dephasing of the collective spin. At time $\tau$, a $\pi$ rotation of the spins is performed in the Bloch sphere, which is equivalent to a time reversal operation, since the phases of the excited and ground state amplitudes get interchanged, i.e., $\mathcal{A}|g\rangle+\mathcal{B} e^{-i \Phi}|e\rangle \rightarrow \mathcal{B} e^{-i \Phi}|g\rangle+\mathcal{A}|e\rangle$, where $\Phi$ is the relative phase between the ground state $|g\rangle$ and the excited state $|e\rangle$. As $\Phi \propto \omega_{a}^{j} \tau$, where $\hbar \omega_{a}^{j}$ is the energy difference between $|e\rangle$ and $|g\rangle$, the subsequent time evolution leads to refocusing of the spins to recover the original coherent superposition state and produce a spin echo at time $2 \tau$.

A range of recent ESR studies have begun to examine increasing the coupling between the spin ensemble and the resonator, for example, to improve spin sensitivity in spectroscopic applications [21,22], or to improve the efficiency of a quantum memories or transducers [1317,23-30]. However, increasing the spin-resonator coupling also introduces the possibility for the emitted echo itself to act as a sufficient perturbing field to drive further evolution of the spins. If the light-matter coupling becomes stronger than the dissipative losses, even the simple and ubiquitous Hahn echo sequence can yield nontrivial spin dynamics which lead to the emission of not just to one, but multiple spin echoes. Such multiple echoes are illustrated in Fig. 1(a) which shows the experimentally observed emission from an inhomogeneously broadened spin ensemble ( $\mathrm{Nd}$ ions in $\mathrm{Y}_{2} \mathrm{SiO}_{5}$ ), subject only to a Hahn echo sequence. (Further details on the experiment are provided in the Supplemental Material [31]). The data reveal the conventional echo signal at time $2 \tau$, followed by additional echoes separated by $\tau$. A similar result was reported recently in Ref. [33], which showed that the secondary echoes were absent when the light-matter coupling was reduced. Indeed, secondary echoes were discerned in the very first microwave spin echo experiment in 1958 [34], where highly doped samples were used to compensate low detector sensitivity. In this Letter we examine the "echo train" phenomenon experimentally and theoretically, with the goal of developing a better understanding of the underlying mechanisms, necessary for the exploitation of strongly coupled resonators and spin ensembles. 


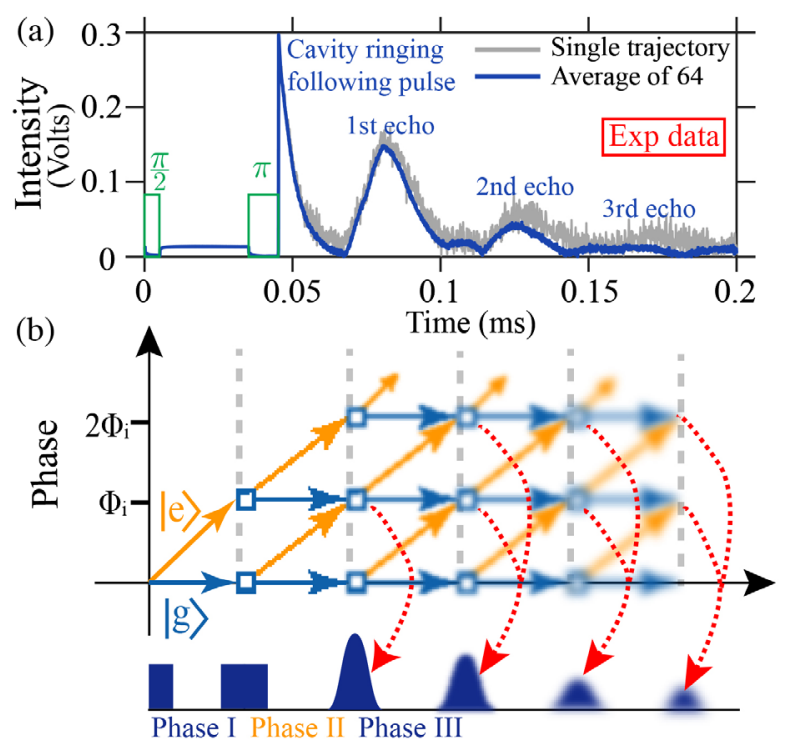

FIG. 1. (a) Experimentally observed emission from an inhomogeneously broadened spin ensemble $\left({ }^{145} \mathrm{Nd}\right.$ ions doped at $200 \mathrm{ppm}$ in a $\mathrm{Y}_{2} \mathrm{SiO}_{5}$ host) in a cavity subject to a conventional Hahn echo sequence with $\tau=30 \mu$ s pulse delay. The spin-cavity coupling exhibits high cooperativity $C=153$ (see the Supplemental Material [31] for full details). For reference, the $\pi / 2$ and $\pi$ pulses are shown in green on a different intensity scale. (b) Schematic of the refocusing mechanism leading to a selfstimulated spin echo train (see text).

Before we present our theoretical results, we offer an intuitive and qualitative description of the dynamics of the spins subjected to imperfect $\pi / 2$ and $\pi$ pulses. Assuming that a spin with frequency $\omega_{a}^{j}$ is initially in the ground state, application of the first pulse excites the spin to a superposition state $\mathcal{A}|g\rangle+\mathcal{B}|e\rangle$. A free evolution for time $\tau$ leads to $\mathcal{A}|g\rangle+\mathcal{B} e^{-i \omega_{a}^{j} \tau}|e\rangle$. A schematic of the above process is shown in Phase I in Fig. 1(b), where blue (orange) corresponds to the phase acquired by the ground (excited) state. The second pulse updates the state of the spin to

$$
\mathcal{A}_{1}|g\rangle+\mathcal{A}_{2}|e\rangle+\mathcal{B}_{1} e^{-i \omega_{a}^{j} \tau}|e\rangle+\mathcal{B}_{2} e^{-i \omega_{a}^{j} \tau}|g\rangle
$$

Here and in the following, the state amplitudes $\mathcal{A}, \mathcal{A}_{m}, \mathcal{B}, \mathcal{B}_{m}$ etc., explore different values that need not be specified for our qualitative discussion. In the schematic in Fig. 1(b), the action of each pulse is shown as bifurcating blue and orange arrows representing the phase evolution for time $\tau$ of the, respectively, ground and excited state amplitudes. The figure also depicts how, after a subsequent time $\tau$, two terms in the time evolved state (underlined below) come in phase:

$$
\mathcal{A}_{1}|g\rangle+\underline{\mathcal{A}_{2} e^{-i \omega_{a}^{j} \tau}|e\rangle}+\mathcal{B}_{1} e^{-2 i \omega_{a}^{j} \tau}|e\rangle+\underline{\mathcal{B}_{2} e^{-i \omega_{a}^{j} \tau}|g\rangle}
$$

This rephasing occurs for all values of the frequency of the spins and leads to the usual Hahn echo.

In case of a perfect $\pi$ refocusing pulse, the state amplitudes $\mathcal{A}_{1}$ and $\mathcal{B}_{1}$ vanish, and the spins accumulate diverging phases in the time following this spin echo. Otherwise, all four terms in (2) contribute to the subsequent evolution of the system, and this is key for the production of further echoes. For large $N$, the Hahn echo pulse may be strong enough to significantly alter the spin states and thus populate a new superposition:

$$
\begin{aligned}
& \left(\mathcal{A}_{11}|g\rangle+\underline{\left.\mathcal{A}_{12}|e\rangle\right)}+e^{-i \omega_{a}^{j} \tau}\left(\underline{\mathcal{C}_{21}|g\rangle}+\underline{\mathcal{C}_{22}|e\rangle}\right)\right. \\
& +e^{-2 i \omega_{a}^{j} \tau}\left(\underline{\mathcal{B}_{11}|g\rangle}+\mathcal{B}_{12}|e\rangle\right) .
\end{aligned}
$$

As can be seen from Fig. 1(b), after a further time $\tau$, the underlined (and doubly underlined) terms come into phase and cause a second echo. The evolution and refocusing may occur also for terms with higher phase arguments, and the process can repeat and lead to multiple self-stimulated echoes.

To assess the validity of our qualitative discussion, we now proceed to investigate whether the spin echo train can be reproduced by a numerical treatment. We begin with a description of the model, followed by the numerical results, demonstrating clear evidence of the self-stimulated spin echo train. We then introduce a simplified analytical model that predicts various shapes of the echo pulse and provide insights into the collective effects involved.

Theoretical model. - We consider $N=10^{10145} \mathrm{Nd}$ spins coupled to a resonator of linewidth $\kappa=2 \pi \times 150 \mathrm{kHz}$ and resonance frequency $\omega_{c}$. The spins are inhomogeneously broadened with transition frequencies $\omega_{a}^{j}$, following a Gaussian distribution with central frequency $2 \pi \times 8 \mathrm{GHz}$ and FWHM of $\Gamma_{\text {inh }}=2 \pi \times 4 \mathrm{MHz}$. An external coherent drive with frequency $\omega_{p}$ and amplitude $F$ is used to apply the two initial pulses. In the frame rotating with the pump frequency, the Hamiltonian $(\hbar=1)$ of the system can be written as

$H=\delta_{c} a^{\dagger} a+\sum_{j=1}^{N}\left[\frac{\delta_{a}^{j}}{2} \sigma_{z}^{j}+g_{j}\left(a \sigma_{+}^{j}+a^{\dagger} \sigma_{-}^{j}\right)\right]+F\left(a+a^{\dagger}\right)$,

where $\delta_{c}=\left(\omega_{c}-\omega_{p}\right)$ and $\delta_{a}^{j}=\left(\omega_{a}^{j}-\omega_{p}\right) \cdot \sigma_{z}^{j}, \sigma_{+}^{j}, \sigma_{-}^{j}$ are the Pauli operators and $a, a^{\dagger}$ are the annihilation and creation operators of the cavity mode, obeying the usual commutation relation $\left[a, a^{\dagger}\right]=1$. For numerical simplicity, we assume $g_{j}=g=2 \pi \times 8 \mathrm{~Hz}$. Despite this weak single spin-cavity coupling, the presence of $10^{10}$ spins significantly enhances the effective coupling. The exact dynamics of the system can be described by the master equation $\dot{\rho}=-i[H, \rho]+\kappa \mathcal{D}[a] \rho+\gamma \sum_{j=1}^{N} \mathcal{D}\left[\sigma_{-}^{j}\right] \rho+\Gamma \sum_{j=1}^{N} \mathcal{D}\left[\sigma_{z}^{j}\right] \rho$. 

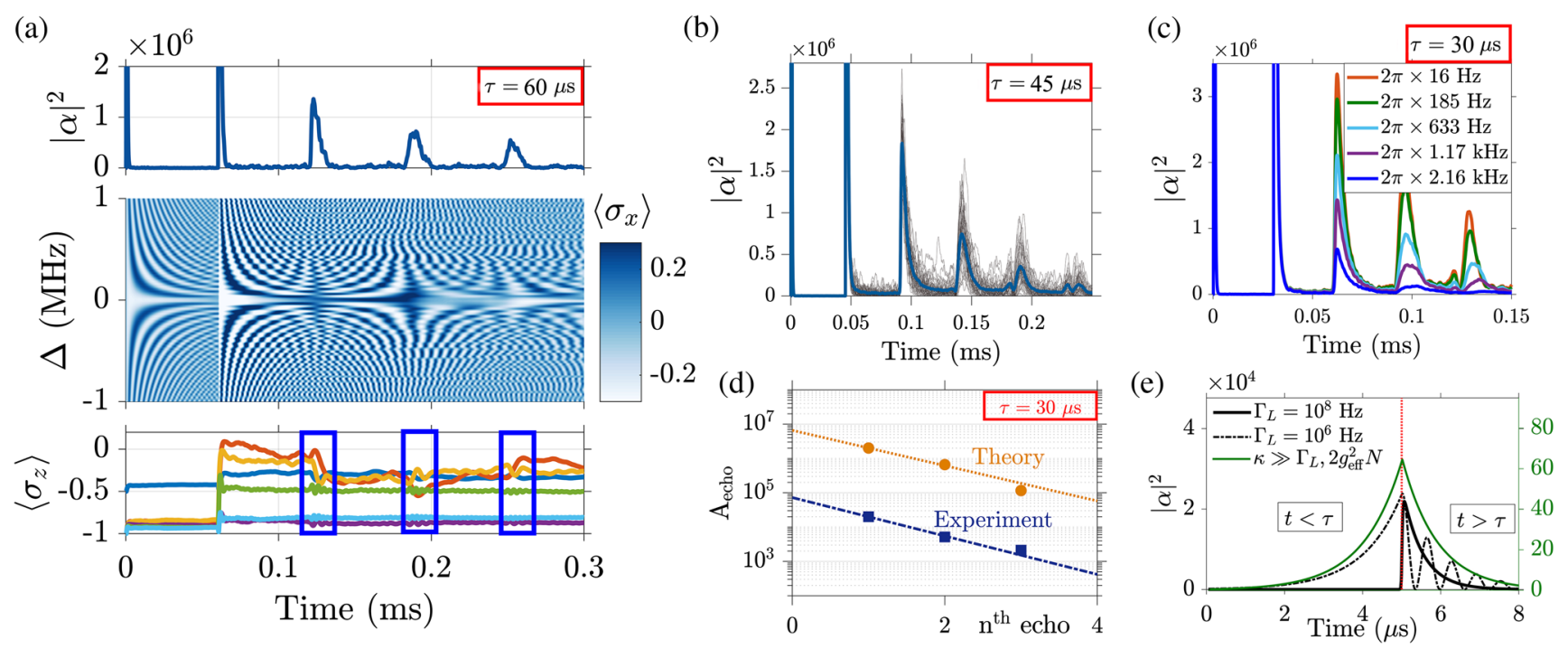

FIG. 2. Numerical results (a) Top: Intracavity photon number $|\alpha|^{2}$ as a function of time for a numerical calculation with $\tau=60 \mu$ s and $\Gamma=2 \pi \times 0.5 \mathrm{kHz}$ (for other parameters, see text). Center: $\left\langle\sigma_{x}\right\rangle$ for the spins in a small detuning region as a function of time. Bottom: $\left\langle\sigma_{z}\right\rangle$ for selected frequency classes. (b) Theoretical results averaged over 45 different samples of the detuning distributions. We consider $\tau=45 \mu \mathrm{s}, \Gamma=2 \pi \times 0.5 \mathrm{kHz}$ and we observe that the echoes change shape over time. (c) Averaged $|\alpha|^{2}$ for $\tau=30 \mu \mathrm{s}$ and different values of the spin dephasing rate $\Gamma$. (d) Emitted number of photons in the echoes as a function of their order of arrival according to theory (dotted) and experiment (dot dashed, arbitrary units). The results are shown for $\tau=30 \mu \mathrm{s}$ and $\Gamma=2 \pi \times 2.5 \mathrm{kHz}$. An exponential fit $y=a e^{-b x}$ reveals a decay rate of $b=2 \pi \times\{6.29 \mathrm{kHz}$ (theory), $6.84 \mathrm{kHz}$ (experiment) $\}>\Gamma$. (e) The first spin echo signal for different values of the Lorentzian inhomogeneous width $\Gamma_{L}$ as computed from Eq. (7), assuming an (overestimated) Holstein-Primakoff spin excitation amplitude $\beta=1$.

The superoperator is defined as $\mathcal{D}[\mathcal{O}] \rho=\mathcal{O} \rho \mathcal{O}^{\dagger}-$ $\frac{1}{2}\left\{\mathcal{O}^{\dagger} \mathcal{O}, \rho\right\}$, for the operators $\mathcal{O}=a, \sigma_{-}^{j}, \sigma_{z}^{j}$. The lifetime $\gamma^{-1}$ of the excited state of the spins is of the order of seconds, and it is a good approximation to neglect spin decay while spin dephasing with a rate $\Gamma \simeq 2 \pi \times 1 \mathrm{kHz}$ plays a significant role on the timescale of interest. Solving the master equation for the full density matrix is impossible, and we treat the model by discretizing the frequency distribution into $N_{k}=10^{5}$ frequency classes following a Gaussian distribution and employ mean field theory for the field and spin raising and lowering operators, assuming factorization of their products, i.e., $\langle A B\rangle \approx\langle A\rangle\langle B\rangle$ [35].

Results.-We apply a strong classical square pulse of amplitude $F=50 \mathrm{GHz}$ from $t_{1}=0.20 \mu$ s to $t_{2}=0.42 \mu \mathrm{s}$, followed by a delay of variable duration $\tau$ and a second pulse between $t_{3}=t_{2}+\tau$ and $t_{4}=t_{3}+0.43 \mu \mathrm{s}$. Any finite pulse area causes some coherent transfer of population between the spin eigenstates and the exact intensity is not crucial for the appearance of the echo as long as the pulses excite the spins by a significant amount.

In Fig. 2(a) we present the dynamics of the spin ensemble for $\tau=60 \mu \mathrm{s}, \Gamma=2 \pi \times 0.5 \mathrm{kHz}$. The top panel shows the square of the cavity field amplitude, i.e., the intracavity photon number, $|\alpha|^{2} \equiv|\langle a\rangle|^{2}$. The first two peaks, which extend beyond the border of the figure, correspond to the two external driving pulses, and we observe that the Hahn echo sequence generates multiple echoes separated by $\tau=60 \mu$ s. To understand this better, we plot $\left\langle\sigma_{x}\right\rangle$ for single spins in different frequency classes close to the resonance in the center panel of Fig. 2(a), where $\Delta=\delta_{a}^{j}-\delta_{c}$. For simplicity, we consider $\omega_{c}=\omega_{p}$ and hence $\Delta=\delta_{a}^{j}$. The refocusing of the spins is characterized by $\left\langle\sigma_{x}\right\rangle$ and $\left\langle\sigma_{y}\right\rangle$ converging to the same finite value for a range of detunings at $2 \tau$ and with reduced strength at later multiples of $\tau$. The lower panel of Fig. 2(a) shows the dynamics of the $z$ component of the spin vector for individual spins in few selected frequency classes. The blue boxes highlight the region where the individual spins get a boost due to the strong mean field, appearing in the cavity when the collective spin refocuses.

In Fig. 2(b) we analyze the shape of the echo signal by averaging multiple trajectories (shown in grey) with randomly sampled Gaussian distributed frequency classes for $\tau=45 \mu \mathrm{s}$ and $\Gamma=2 \pi \times 0.5 \mathrm{kHz}$. A consistent plot of $|\alpha|^{2}$ demands many closely spaced frequency classes $\left(N_{k} \gg 10^{5}\right)$, which is a challenging task even with a mean field approximation. Averaging multiple realizations results in a more consistent data and $|\alpha|^{2}$ averaged over 45 realizations is shown with the bold curve. The rate $\Gamma$, which takes into account dephasing due to different mechanisms such as mutual interactions of the spins, spectral diffusion due to crystal deformation and phonon induced energy shifts, leads to vanishing of $\left\langle\sigma_{x}\right\rangle$ and $\left\langle\sigma_{y}\right\rangle$ and breakdown of refocusing at large $t$. The separation between the echoes increases with increasing $\tau$, as evident from Figs. 2(a)-2(c) 
and the phase of the output cavity field due to the echoes at $t=2 \tau, 3 \tau, \ldots$ depends on the phase of the refocusing pulse [31].

Figure 2(c) shows how an increase of the spin dephasing rate $\Gamma$ causes a flattening of the shape of the echoes for $\tau=30 \mu$ s. It also explains the symmetric shape of the echo observed in the experiments with the dephasing rate $\Gamma=$ $2 \pi \times 2.5 \mathrm{kHz}$ [Fig. 1(a)] and in Ref. [31].

Following Ref. [33], in Fig. 2(d), we plot the emitted photon number in each echo $A_{\text {echo }}=\int_{\text {echo }}|\alpha|^{2} \kappa d t$ as a function of its order of emission from both experiments and numerical calculations with $\tau=30 \mu \mathrm{s}$ and $\Gamma=2 \pi \times 2.5 \mathrm{kHz}$. The experimental intensity data is not absolutely calibrated and has been displaced (in the $y$ direction) by an arbitrary amount in the plot for better comparison with theory. An exponential fit, which is independent of the arbitrary displacement, reveals that the echoes decay faster than the spin dephasing rate $\Gamma$ [see also Figs. S1(a)-S1(d) in [31]] while we observe an excellent agreement between theory $(2 \pi \times 6.29 \mathrm{kHz})$ and experiment $(2 \pi \times 6.84 \mathrm{kHz})$. We attribute this faster decay to the incomplete refocusing of the spins by the weakening pulses associated with the observed distortion and lengthening of the pulse shapes and the reduction in their amplitude. The inhomogeneous spincavity coupling may also affect the effective nonlinear dynamics.

We can understand the shape of the first Hahn echo observed in Fig. 2(b) by a simple analytical model. Rather than solving the complete spin dynamics analytically, we assume that a perfect $\pi / 2$ and $\pi$ pulse have been applied at $t=-\tau$ and $t=0$, respectively, to all the spins. This implies that right after $t=0$, the spin excited states have acquired a phase of $\exp \left(i \Delta_{j} \tau\right)$ with respect to the spin ground states. Since the spin excited states evolve as $\exp \left(-i \Delta_{j} t\right)$, they come in phase at $t=\tau$. To model the resulting Hahn echo pulse shape, we employ the Holstein-Primakoff approximation [36] and treat all spins as harmonic oscillators prepared in a coherent state of complex amplitude $\beta \exp \left(i \Delta_{j} \tau\right)$ at $t=0$. Assuming $\Gamma=0$, the mean field equations for the intracavity field operator and the spin lowering operator take the following form in the frequency domain

$$
\begin{aligned}
& -i \omega\langle\tilde{a}(\omega)\rangle=-\frac{\kappa}{2}\langle\tilde{a}(\omega)\rangle-i \sum_{j}^{N} g_{j}\left\langle\tilde{\sigma}_{j}(\omega)\right\rangle, \\
& -i \omega\left\langle\tilde{\sigma}_{j}(\omega)\right\rangle=-\left(\gamma+i \Delta_{j}\right)\left\langle\tilde{\sigma}_{j}(\omega)\right\rangle-i g_{j}\langle\tilde{a}(\omega)\rangle+\frac{\beta}{\sqrt{2 \pi}} e^{i \Delta_{j} \tau} .
\end{aligned}
$$

The above equations can be formally solved, which yields

$$
\langle\tilde{a}(\omega)\rangle=\frac{\frac{-i}{\sqrt{2 \pi}} \sum_{j}^{N} \beta g_{j} e^{i \Delta_{j} \tau} /\left(\gamma+i \Delta_{j}-i \omega\right)}{\frac{\kappa}{2}-i \omega+\sum_{j}^{N} g_{j}^{2} /\left(\gamma+i \Delta_{j}-i \omega\right)} .
$$

Since the detunings $\Delta_{j}$ have a continuous distribution, the summation sign can be replaced by an integral in the limit of large $N$, i.e., $\sum_{j}^{N} \cdot \rightarrow N \int f(\Delta) d \Delta . f(\Delta)=\left[\left(\Gamma_{L} / 2 \pi\right) /\right.$ $\left.\left(\Delta^{2}+\Gamma_{L}^{2} / 4\right)\right]$ for a Lorentzian distribution and $f(\Delta)=$ $\left(1 / \sqrt{2 \pi} \Gamma_{G}\right) \exp \left(-\Delta^{2} / 2 \Gamma_{G}^{2}\right)$ for a Gaussian distribution, where $\Gamma_{L}$ and $\Gamma_{G} \sqrt{8 \ln (2)}$ are their full width at half maxima (FWHM) respectively. In principle, Eq. (6) can be solved for a Gaussian distribution [37], however, it is not possible to obtain a general analytical expression for $\langle a(t)\rangle$, and we shall therefore provide analytical results for Lorentzian distributions. Assuming that the inhomogeneity in the light-matter coupling is weak, such that $g_{j} \rightarrow g_{\text {eff }}=\sqrt{(1 / N) \sum_{j}^{N}\left|g_{j}^{2}\right|}$, and putting $\gamma=0$, the above equation can be transformed to the time domain, leading to

$$
\begin{aligned}
& \left\langle a(\boldsymbol{t}\langle\boldsymbol{\tau})\rangle=\left(\frac{2 i \beta N \Gamma_{L}}{\sqrt{2 \pi}}\right)\left[\frac{e^{-\Gamma_{L}(\tau-t) / 2}}{-\Gamma_{L}^{2}-\kappa \Gamma_{L}-2 g_{\mathrm{eff}}^{2} N}\right],\right. \\
& \langle a(\boldsymbol{t}>\boldsymbol{\tau})\rangle=\left(\frac{2 i \beta N \Gamma_{L}}{\sqrt{2 \pi}}\right)\left[\frac{4 e^{\Sigma_{-}(\tau-t)}}{\Theta_{+}}-\frac{4 e^{\Sigma_{+}(\tau-t)}}{\Theta_{-}}\right],
\end{aligned}
$$

where $\zeta^{2}=16 g_{\mathrm{eff}}^{2} N-\left(\Gamma_{L}-\kappa\right)^{2}, \Sigma_{ \pm}=\left(\Gamma_{L}+\kappa \pm i \zeta\right) / 4$, and $\Theta_{ \pm}=\zeta\left(3 i \Gamma_{L}+i \kappa \pm \zeta\right)$. We use Eq. (7) to plot $|\alpha|^{2}$ in Fig. 2(e) for different values of $\Gamma_{L}$. It is evident from Eq. (7) that for $t<\tau$, the refocusing of the spins lead to $\langle a\rangle \propto e^{-\Gamma_{L}(\tau-t) / 2}$ for any choice of parameters. However, for $t>\tau$, the choice of parameters governs the shape of the decaying echo signal. When $\Gamma_{L}$ is sufficiently large, i.e., $\left(\Gamma_{L}-\kappa\right)>4 g_{\mathrm{eff}} \sqrt{N}, \Sigma_{ \pm}$is real and $\langle a\rangle$ is proportional to $\left(2 e^{-\kappa(t-\tau) / 2}-e^{-\Gamma_{L}(t-\tau) / 2}\right)$. If $\Gamma_{L}>\kappa$ (bold blue curve), the second term vanishes rapidly and the shape of the pulse is predominantly dictated by the decay of the field out of the cavity, i.e., $\langle a\rangle \propto e^{-\kappa(t-\tau) / 2}$. When the collective coupling $g_{\text {eff }} \sqrt{N}$ is sufficiently large, $\zeta$ becomes real and exhibits damped oscillations (dot-dashed orange curve) due to the coherent exchange of energy between the spins and the cavity mode. On the other hand, when the spins are coupled to a bad cavity, i.e., $\kappa \gg \Gamma_{L}, 2 g_{\text {eff }}^{2} N$, the echo shape becomes symmetric about $t=\tau$ (green bold curve, right-hand axis). Note that the above analysis assumes $\gamma=\Gamma=0$, and as we observed above, the echo pulses from a good cavity may also be symmetric if $\Gamma$ is large.

Conclusion.-To conclude, we have observed and characterized the appearance of echo trains after the simple Hahn echo sequence, and we have argued and shown by theoretical calculations that they are due to spin refocusing by previous echo pulses. Our mean field calculations show that the echo field amplitude is indeed strong enough to appreciably alter the individual spin states, and hence cause their refocusing at later times. Due to the finite duration of the pulse, its refocusing effect on differently detuned spin components is more complex, and the later echo is weaker 
and has a more complex structure. Note that the mechanism leads to even later echoes which may all carry contributions from the refocusing by both the most recent and earlier echo pulses. Our numerical results reproduce most of the qualitative features observed in the experiments and show excellent quantitative agreement with the observed decay of the integrated echo pulse intensities.

Given the complexity of the model, it is difficult to analytically predict the shape of secondary echoes. Future efforts shall be devoted to understand the scaling laws that govern the gradual reduction of the self-stimulated echo amplitudes, and how these depend on the spin and cavity parameters. It is evident from the present study that in addition to the cavity linewidth and the spin dephasing rates [33], this decay will also depend nonlinearly on the intensity of the echoes, e.g., due to the incomplete refocusing by previous echoes, and the total number of spins effectively coupled to the resonator. Other factors such as the distribution of detunings and coupling strengths may offer varying contribution to the precise behavior of this decay. Although the secondary echoes studied here may constitute an unavoidable feature to be mitigated in quantum memory or transducer protocols involving spin ensembles, they may also offer opportunities in ESR spectroscopy. Aside from the potential to improve signal-to-noise by averaging multiple echoes in one train, these secondary echoes could provide an efficient route to obtaining information on loss rates in the system [33] or reveal spectroscopic information, for example in more complex spin systems with unresolved hyperfine couplings. The understanding and theoretical framework that we present here forms a basis to explore such opportunities in more detail.

K.D. and K. M. acknowledge support from the European Union FETFLAG program, Grant No. 820391 (SQUARE) and the Villum Foundation. G. D. and J. J. L. M. acknowledge support from the Engineering and Physical Sciences Research Council (EPSRC) through the Centre for Doctoral Training in Delivering Quantum Technologies (Grant No. EP/L015242/1) and Quantum Engineering of Solid-state Technologies (Grant No. EP/ N015118/1), as well as from the Horizon 2020 research and innovation programme through Grant Agreement No. 771493 (LOQO-MOTIONS).

*kamanasish.debnath@phys.au.dk

[1] W. V. Smith, P. P. Sorokin, I. L. Gelles, and G. J. Lasher, Electron-spin resonance of nitrogen donors in diamond, Phys. Rev. 115, 1546 (1959).

[2] C. Eichler, A. J. Sigillito, S. A. Lyon, and J. R. Petta, Electron Spin Resonance at the Level of $10^{4}$ Spins Using Low Impedance Superconducting Resonators, Phys. Rev. Lett. 118, 037701 (2017).

[3] N. Aslam, M. Pfender, P. Neumann, R. Reuter, A. Zappe, F. Fávaro de Oliveira, A. Denisenko, H. Sumiya, S. Onoda,
J. Isoya, and J. Wrachtrup, Nanoscale nuclear magnetic resonance with chemical resolution, Science 357, 67 (2017).

[4] C. H. Pennington and V. A. Stenger, Nuclear magnetic resonance of $\mathrm{C}_{60}$ and fulleride superconductors, Rev. Mod. Phys. 68, 855 (1996).

[5] G. E. Merz, P. P. Borbat, A. R. Muok, M. Srivastava, D. N. Bunck, J. H. Freed, and B. R. Crane, Site-specific incorporation of a $\mathrm{Cu}^{2+}$ spin label into proteins for measuring distances by pulsed dipolar electron spin resonance spectroscopy, J. Phys. Chem. B 122, 9443 (2018).

[6] A. G. Palmer, Enzyme dynamics from NMR spectroscopy, Acc. Chem. Res. 48, 457 (2015).

[7] T. W.-M. Fan and A. N. Lane, Applications of NMR spectroscopy to systems biochemistry, Prog. Nucl. Magn. Reson. Spectrosc. 92-93, 18 (2016).

[8] J. Niklas and O. G. Poluektov, Charge transfer processes in OPV materials as revealed by EPR spectroscopy, Adv. Energy Mater. 7, 1602226 (2017).

[9] B. L. Edlow, A. Mareyam, A. Horn, J. R. Polimeni, T. Witzel, M. D. Tisdall, J. C. Augustinack, J. P. Stockmann, B. R. Diamond, A. Stevens, L. S. Tirrell, R. D. Folkerth, L. L. Wald, B. Fischl, and A. Kouwe, 7 Tesla MRI of the ex vivo human brain at 100 micron resolution, Sci. Data 6, 244 (2019).

[10] N. A. Kurnit, I. D. Abella, and S. R. Hartmann, Observation of a Photon Echo, Phys. Rev. Lett. 13, 567 (1964).

[11] W. G. Breiland, C. B. Harris, and A. Pines, Optically Detected Electron Spin Echoes and Free Precession in Molecular Excited States, Phys. Rev. Lett. 30, 158 (1973).

[12] E. L. Hahn, Spin echoes, Phys. Rev. 80, 580 (1950).

[13] H. Wu, R. E. George, J. H. Wesenberg, K. Mølmer, D. I. Schuster, R. J. Schoelkopf, K. M. Itoh, A. Ardavan, J. J. L. Morton, and G. A. D. Briggs, Storage of Multiple Coherent Microwave Excitations in an Electron Spin Ensemble, Phys. Rev. Lett. 105, 140503 (2010).

[14] B. Julsgaard and K. Mølmer, Fundamental limitations in spin-ensemble quantum memories for cavity fields, Phys. Rev. A 88, 062324 (2013).

[15] B. Julsgaard, C. Grezes, P. Bertet, and K. Mølmer, Quantum Memory for Microwave Photons in an Inhomogeneously Broadened Spin Ensemble, Phys. Rev. Lett. 110, 250503 (2013).

[16] C. Grezes, B. Julsgaard, Y. Kubo, M. Stern, T. Umeda, J. Isoya, H. Sumiya, H. Abe, S. Onoda, T. Ohshima, V. Jacques, J. Esteve, D. Vion, D. Esteve, K. Mølmer, and P. Bertet, Multimode Storage and Retrieval of Microwave Fields in a Spin Ensemble, Phys. Rev. X 4, 021049 (2014).

[17] C. Simon et al., Quantum memories, Eur. Phys. J. D 58, 1 (2010).

[18] J. R. Maze, P. L. Stanwix, J. S. Hodges, S. Hong, J. M. Taylor, P. Cappellaro, L. Jiang, M. V. G. Dutt, E. Togan, A. S. Zibrov, A. Yacoby, R. L. Walsworth, and M. D. Luki, Nanoscale magnetic sensing with an individual electronic spin in diamond, Nature (London) 455, 644 (2008).

[19] A. Bienfait, P. Campagne-Ibarcq, A. H. Kiilerich, X. Zhou, S. Probst, J. J. Pla, T. Schenkel, D. Vion, D. Esteve, J. J. L. Morton, K. Moelmer, and P. Bertet, Magnetic Resonance with Squeezed Microwaves, Phys. Rev. X 7, 041011 (2017).

[20] N. Bar-Gill, L. M. Pham, C. Belthangady, D. Le Sage, P. Cappellaro, J. R. Maze, M. D. Lukin, A. Yacoby, and 
R. Walsworth, Suppression of spin-bath dynamics for improved coherence of multi-spin-qubit systems, Nat. Commun. 3, 858 (2012).

[21] A. Bienfait, J. J. Pla, Y. Kubo, M. Stern, X. Zhou, C. C. Lo, C. D. Weis, T. Schenkel, M. L. W. Thewalt, D. Vion, D. Esteve, B. Julsgaard, K. Mølmer, J. J. L. Morton, and P. Bertet, Reaching the quantum limit of sensitivity in electron spin resonance, Nat. Nanotechnol. 11, 253 (2016).

[22] S. Probst, A. Bienfait, P. Campagne-Ibarcq, J. J. Pla, B. Albanese, J. F. Da Silva Barbosa, T. Schenkel, D. Vion, D. Esteve, K. Mølmer, J. J. L. Morton, R. Heeres, and P. Bertet, Inductive-detection electron-spin resonance spectroscopy with 65 spins/ $\sqrt{\mathrm{Hz}}$ sensitivity, Appl. Phys. Lett. 111, 202604 (2017).

[23] G. Dold, C. W. Zollitsch, J. O’Sullivan, S. Welinski, A. Ferrier, P. Goldner, S. E. de Graaf, T. Lindström, and J. J. L. Morton, High-Cooperativity Coupling of a Rare-Earth Spin Ensemble to a Superconducting Resonator Using Yttrium Orthosilicate as a Substrate, Phys. Rev. Applied 11, 054082 (2019).

[24] N. Shammah, S. Ahmed, N. Lambert, S. De Liberato, and F. Nori, Open quantum systems with local and collective incoherent processes: Efficient numerical simulations using permutational invariance, Phys. Rev. A 98, 063815 (2018).

[25] R. Amsüss, Ch. Koller, T. Nöbauer, S. Putz, S. Rotter, K. Sandner, S. Schneider, M. Schramböck, G. Steinhauser, H. Ritsch, J. Schmiedmayer, and J. Majer, Cavity QED with Magnetically Coupled Collective Spin States, Phys. Rev. Lett. 107, 060502 (2011).

[26] K. Sandner, H. Ritsch, R. Amsüss, Ch. Koller, T. Nöbauer, S. Putz, J. Schmiedmayer, and J. Majer, Strong magnetic coupling of an inhomogeneous nitrogen-vacancy ensemble to a cavity, Phys. Rev. A 85, 053806 (2012).

[27] R. H. Dicke, Coherence in spontaneous radiation processes, Phys. Rev. 93, 99 (1954).
[28] K. Debnath, Y. Zhang, and K. Mølmer, Collective dynamics of inhomogeneously broadened emitters coupled to an optical cavity with narrow linewidth, Phys. Rev. A 100, 053821 (2019).

[29] B. Kraus, W. Tittel, N. Gisin, M. Nilsson, S. Kröll, and J. I. Cirac, Quantum memory for nonstationary light fields based on controlled reversible inhomogeneous broadening, Phys. Rev. A 73, 020302(R) (2006).

[30] I. Diniz, S. Portolan, R. Ferreira, J. M. Gérard, P. Bertet, and A. Auffèves, Strongly coupling a cavity to inhomogeneous ensembles of emitters: Potential for long-lived solid-state quantum memories, Phys. Rev. A 84, 063810 (2011).

[31] See the Supplemental Material at http://link.aps.org/ supplemental/10.1103/PhysRevLett.125.137702 for experimental data, which includes Ref. [32].

[32] M. Xu, D. A. Tieri, E. C. Fine, J. K. Thompson, and M. J. Holland, Synchronization of Two Ensembles of Atoms, Phys. Rev. Lett. 113, 154101 (2014).

[33] S. Weichselbaumer, C. W. Zollitsch, M. S. Brandt, R. Gross, and H. Huebl, companion Letter, Echo trains in pulsed electron spin resonance of a strongly coupled spin ensemble, Phys. Rev. Lett. 125, 137701 (2020).

[34] J. P. Gordon and K. D. Bowers, Microwave Spin Echoes from Donor Electrons in Silicon, Phys. Rev. Lett. 1, 368 (1958).

[35] K. Debnath, Y. Zhang, and K. Mølmer, Lasing in the superradiant crossover regime, Phys. Rev. A 98, 063837 (2018).

[36] T. Holstein and H. Primakoff, Field dependence of the intrinsic domain magnetization of a ferromagnet, Phys. Rev. 58, 1098 (1940).

[37] B. Julsgaard and K. Mølmer, Dynamical evolution of an inverted spin ensemble in a cavity: Inhomogeneous broadening as a stabilizing mechanism, Phys. Rev. A 86, 063810 (2012). 